SANTOS APG; VIANA TVA; SOUSA GG; Ó LMG; AZEVEDO BM; SANTOS AM. 2014. Produtividade e qualidade de frutos do meloeiro em função de tipos e doses de biofertilizantes. Horticultura Brasileira 32: 409-416. DOI - http://dx.doi.org/10.1590/S0102-053620140000400007

\title{
Produtividade e qualidade de frutos do meloeiro em função de tipos e doses de biofertilizantes
}

\author{
Ana PG Santos; Thales VA Viana; Geocleber G Sousa; Laís M Gomes-do-Ó; Benito M Azevedo; Ailton \\ M Santos \\ UFC, C. Postal 12168,60455-970 Fortaleza-CE; apgsr@hotmail.com; thales@ufc.br; sousamsa@yahoo.com.br; benitoazevedo@ \\ hotmail.com; ailtonagr@yahoo.com.br
}

\begin{abstract}
RESUMO
O melão (Cucumis melo) apresenta elevados gastos com adubos minerais, evidenciando a possibilidade da utilização de produtos alternativos como os biofertilizantes. Este experimento teve como objetivo avaliar a produtividade e a pós-colheita da cultura do melão cultivar Mirage seguimento Harper, submetida a doses e tipos de biofertilizantes na presença e ausência de adubação mineral. O delineamento experimental foi em blocos casualizados no arranjo fatorial $4 \times 2+2$, referentes a quatro doses de biofertilizantes $(0,5$, $1,0,1,5$ e 2,0 L/semana), dois tipos de biofertilizantes líquidos (misto de fermentação aeróbica e bovino simples de fermentação anaeróbica) com dois tratamentos adicionais: controle e adubação mineral recomendada. As variáveis analisadas foram produtividade, massa média de frutos, diâmetro de frutos, sólidos solúveis, acidez titulável, firmeza, espessura da polpa e cavidade da polpa. O biofertilizante misto mostrou-se mais eficiente do que o bovino na maioria das variáveis analisadas. A maior produtividade do meloeiro (32,62 t/ha) foi alcançada com a dose de 1,08 L/planta/semana para o biofertilizante misto e com 1,41 L/planta/semana para o bovino $(25,87 \mathrm{t} / \mathrm{ha})$. O biofertilizante misto e bovino com a dose $2,0 \mathrm{~L} /$ planta/semana foi melhor que o controle e a adubação mineral para espessura e cavidade de polpa.
\end{abstract}

Palavras chave: Cucumis melo, fertilizante orgânico, pós-colheita, sólidos solúveis.

\begin{abstract}
Yield and quality of melon fruits depending on types and doses of biofertilizers

Melon (Cucumis melo) has a high demand of mineral fertilization, suggesting the possibility of using alternative products such as biofertilizers. In this experiment we evaluated the yield and postharvest of the melon crop, cv. Mirage (Harper), subjected to different doses (and types) of biofertilizers. The experimental design was randomized blocks in a $4 \times 2+2$ factorial arrangement, with treatments constituted of four doses of biofertilizers $(0.5$, 1.0, 1.5 and $2.0 \mathrm{~L} /$ week), two types of liquid biofertilizers (mixed aerobically fermented biofertilizer and pure anaerobically fermented bovine biofertilizer) with two additional treatments: control and recommended mineral fertilization. We analyzed yield, average fruit weight, fruit diameter, soluble solids, titratable acidity, firmness, pulp thickness and central cavity. The mixed biofertilizer was more efficient than pure bovine biofertilizer for most of the analyzed variables. The highest melon yield (32.62 t/ha) was achieved with a dose of $1.08 \mathrm{~L} /$ plant/week for mixed biofertilizer and $1.41 \mathrm{~L} /$ plant/ week for pure bovine biofertilizer $(25.87 \mathrm{t} / \mathrm{ha})$. Both the mixed biofertilizer and the pure bovine biofertilizer, at the rate (dose) of $2.0 \mathrm{~L} / \mathrm{plant} /$ week were better than the control and better than mineral fertilization by the criterion of pulp thickness and central cavity.
\end{abstract}

Keywords: Cucumis melo, organic fertilizer, postharvest, soluble solids.

(Recebido para publicação em 22 de maio de 2013; aceito em 22 de agosto de 2014)

(Received on May 22, 2013; accepted on August 22, 2014)

$\mathrm{O}$ melão (Cucumis melo) é originário de regiões tropicais, desenvolve-se melhor em temperaturas elevadas, o que favorece alta produtividade $\mathrm{e}$ frutos de excelente qualidade (Melo et al., 2012). É uma olerícola de relevante importância socioeconômica para o Nordeste brasileiro, em razão do seu alto valor comercial, tanto para o mercado interno, quanto para exportação (IBGE, 2012). Os dados mais recentes demonstram que a produção de melão no mundo é de cerca de 575 mega toneladas, sendo que o Brasil ocupa a décima posição (FAO, 2012).

Nas últimas décadas, vêm sendo utilizadas como alternativa econômica e ambiental, fontes orgânicas em cultivos agrícolas, na substituição parcial ou total de fertilizantes minerais. Nesse sentido, uma das possibilidades para se reduzir o emprego de insumos sintéticos aos solos e às plantas é a utilização de esterco líquido, como os biofertilizantes (Cavalcante et al., 2007).

O biofertilizante é um adubo orgânico líquido produzido em meio aeróbico ou anaeróbico a partir de uma mistura de material orgânico (esterco fresco) e água (Penteado, 2007). Segundo Silva \& Mendonça (2007), a aplicação de produtos orgânicos na agricultura é importante pela diversidade dos nutrientes minerais e pela ação positiva de ativador enzimático do metabolismo vegetal.

Alguns biofertilizantes vêm sendo testados com o intuito de se chegar a uma formulação e a uma elaboração ideal, objetivando-se disponibilizar o máximo de nutrientes para as plantas. Mesquita et al. (2007) avaliaram a produtividade e qualidade de frutos do 
mamoeiro em função de tipos e doses de biofertilizantes (comum e enriquecido) e concluíram que o mamoeiro produz frutos compatíveis às exigências do mercado consumidor sob fertilização do solo em cobertura com biofertilizantes. Por outro lado, Santos et al. (2012), investigando a qualidade de frutos de abóbora em função de doses de biofertilizante, verificaram que o teor de sólidos solúveis na polpa dos frutos foi maior na testemunha, sem biofertilizante.

Neste contexto, a utilização de biofertilizante misto proporcionará maior produtividade e melhor qualidade de frutos de meloeiro em relação à adubação química e ao controle. Este experimento teve como objetivo avaliar a produtividade e a pós-colheita da cultura do melão cultivar Mirage seguimento Harper, submetida a doses e tipos de biofertilizantes na presença e ausência de adubação mineral.

\section{MATERIAL E MÉTODOS}

O experimento foi conduzido na área experimental da Universidade Federal do Ceará, município de Fortaleza-CE (034' 'S, 38 33'O, 19,6 m de altitude). O clima da região, segundo a classificação de Köppen, é do tipo Aw', tropical chuvoso, com temperaturas elevadas e estação chuvosa predominante no outono.

Os tratamentos foram distribuídos em blocos casualizados no arranjo fatorial $2 \times 4+2$, referentes a dois tipos de biofertilizantes líquidos ( $\mathrm{B}=$ biofertilizante misto, com estercos bovino e de ave com fermentação aeróbica; B2= biofertilizante bovino com fermentação anaeróbica), quatro doses de biofertilizante $(2,0 ; 1,5 ; 1,0$ e $0,5 \mathrm{~L} /$ semana) e dois tratamentos adicionais \{um com adubação mineral (CM), recomendada por Terceiro Neto et al. (2012) e um controle $(\mathrm{CO})$ sem adubação (solo + composto orgânico)\}.

A área total ocupada pelo experimento foi de $17 \times 10,5 \mathrm{~m}$ onde foram instalados 160 baldes plásticos, com volume individual de $35 \mathrm{~L}$. O enchimento dos mesmos constituiu-se de uma camada de $5 \mathrm{~L}$ com brita e outra de $5 \mathrm{~L}$ com areia, o restante $(25 \mathrm{~L})$ foi constituído de um substrato formado por uma mistura do solo local (de 0 a $20 \mathrm{~cm}$ ), classificado como Argissolo Vermelho amarelo de textura franco argilo arenosa (Embrapa, 2006), com um composto orgânico sólido Bioadubos da empresa Solo Fértil na proporção de 4:1. Os atributos químicos do substrato foram $\mathrm{N}=1,76 ; \mathrm{Ca}^{2+}=3,6 ; \mathrm{K}^{+}=0,78 ; \mathrm{Mg}^{2+}=$ 3,$4 ; \mathrm{Na}^{+}=0,5 ; \mathrm{H}^{+}+\mathrm{Al}^{3+}=0,17 ; \mathrm{SB}=8,3$; $\mathrm{CTC}=8,45 \mathrm{cmol}_{\mathrm{c}} / \mathrm{dm}^{3} ; \mathrm{MO}=28,24(\mathrm{~g} /$ $\mathrm{kg}) ; \mathrm{V}=98 \% ; \mathrm{P}=305$ (mg/dm); $\mathrm{pH}=7,1$.

A densidade do substrato foi de 1,3 $\mathrm{kg} / \mathrm{dm}^{3}$. A cultivar de meloeiro utilizada foi o Mirage seguimento Harper, Cantaloupe, que tem maior durabilidade pós-colheita. As mudas foram formadas em bandejas de poliestireno com 128 células preenchidas com vermiculita e transferidas para os vasos seis dias após a germinação.

No preparo do biofertilizante misto com fermentação aeróbica utilizaram-se tanques, colocando neles os ingredientes: $100 \mathrm{~L}$ de esterco bovino, $30 \mathrm{~L}$ de esterco de galinha, $5 \mathrm{~L}$ de cinza e água na proporção 1:1. Deixou-se fermentar a mistura de forma aeróbica durante 10 dias, fez-se o revolvimento diário para melhor aeração, sendo ainda utilizado um acelerador de fermentação, o PT-4-O: concentrado de radical carboxílico orgânico e composto de nitrogênio bioativo, sintetizados em laboratório, além de fósforo orgânico fixado em molécula microcelulose.

Já no preparo do biofertilizante bovino com fermentação anaeróbica foram utilizados apenas esterco bovino e água na proporção $1: 1$, com base no volume durante 30 dias. Para se obter o sistema anaeróbico, a mistura foi colocada em recipientes plásticos com capacidade para 240 L deixando-se um espaço vazio de 15 a $20 \mathrm{~cm}$ no seu interior e fechada hermeticamente. Na tampa foi adaptada uma mangueira com a outra extremidade mergulhada num recipiente com água na altura de $20 \mathrm{~cm}$, para a saída de gases (Santos, 1992).

O sistema de irrigação localizada utilizado na condução do experimento foi do tipo gotejamento autocompensante. A instalação do sistema de irrigação foi iniciada com a montagem da linha principal, constituída por uma tubulação de PVC com $5 \mathrm{~cm}$ de diâmetro e $12 \mathrm{~m}$ de comprimento, e do cabeçal de controle composto por manômetro de glicerina e filtro de disco. As linhas de derivação foram compostas de mangueira de polietileno com $2,5 \mathrm{~cm}$ de diâmetro. No início de cada linha de derivação instalou-se um registro, que foi utilizado para o controle da pressão do sistema de irrigação. Nas linhas secundárias instalaram-se em cada vaso um gotejador, autocompensante com vazão de $8 \mathrm{~L} / \mathrm{h}$. A lâmina de irrigação aplicada diariamente foi quantificada a partir da evaporação medida em um tanque classe "A".

Os biofertilizantes constituídos, após o período pré-estabelecido para a fermentação, foram aplicados à cultura por fertilização manual. As biofertilizações iniciaram-se após o transplantio, em conformidade com os tratamentos, durante 10 semanas. As características químicas do biofertilizante misto foram: 406 mg/L de N; 837,26 mg/L de P; 1620 $\mathrm{mg} / \mathrm{L}$ de $\mathrm{K} ; 314,17 \mathrm{mg} / \mathrm{L}$ de Ca; 801,23 $\mathrm{mg} / \mathrm{L}$ de $\mathrm{Mg} ; 314,17$ mg/L de S; 1275 $\mathrm{mg} / \mathrm{L}$ de $\mathrm{Na} ; 89,16 \mathrm{mg} / \mathrm{L}$ de Fe; 0,74 $\mathrm{mg} / \mathrm{L}$ de $\mathrm{Cu} ; 26,42 \mathrm{mg} / \mathrm{L}$ de $\mathrm{Zn} ; 33,53$ $\mathrm{mg} / \mathrm{L}$ de $\mathrm{Mn}$. Por outro lado, as características químicas do biofertilizante bovino foram; $266 \mathrm{mg} / \mathrm{L}$ de $\mathrm{N} ; 518,76$ $\mathrm{mg} / \mathrm{L}$ de P; $1215 \mathrm{mg} / \mathrm{L}$ de K; 172,97 mg/Lde Ca; 3044,6 mg/Lde Mg; 172,87 $\mathrm{mg} / \mathrm{L}$ de $\mathrm{S} ; 380 \mathrm{mg} / \mathrm{Lde} \mathrm{Na}$ 47,99 mg/L de Fe; 0,22 mg/L de $\mathrm{Cu} ; 16,09 \mathrm{mg} / \mathrm{L}$ de $\mathrm{Zn} ; 10,05$ mg/L de Mn.

Para atender às exigências nutricionais das plantas de melão durante o ciclo adotou-se a recomendação máxima da adubação química fornecida por Terceiro Neto et al. (2012) correspondente a: $127 \mathrm{~kg} / \mathrm{ha}$ de $\mathrm{N}, 176 \mathrm{~kg} /$ ha de $\mathrm{P}_{2} \mathrm{O}_{5}$ e 242 $\mathrm{kg} /$ ha de $\mathrm{K}_{2} \mathrm{O}$. Como referência, para um estande de 10.000 plantas (espaçamento de $1 \times 1 \mathrm{~m}$ ) a dosagem máxima recomenda por planta no ciclo seria de: $12,7 \mathrm{~g} \mathrm{~N} ; 17,6 \mathrm{~g} \mathrm{P}_{2} \mathrm{O}_{5}$ e 24,2 g de $\mathrm{K}_{2} \mathrm{O}$. Não houve aplicação de calcário nem de micronutrientes.

A partir das análises químicas do substrato e dos biofertilizantes e das estimativas de fornecimento total dos nutrientes procurou-se fornecer no tratamento com maior dose a recomendação máxima para N, P e K. Após estimativas, adotou-se como dose máxima 2 $\mathrm{L} /$ planta/semana, para tentar suprir a 
recomendação para o $\mathrm{N}, \mathrm{P}$ e K.

O substrato forneceu apenas: 0,24; 0,$30 ; 0,30 \mathrm{~g} / \mathrm{kg}$ de NPK, que ao multiplicar-se pela massa de solo $(32,55 \mathrm{~kg} /$ $\mathrm{dm}^{3}$ ), obteve-se 7,81;9,76;9,76 g de $\mathrm{N}, \mathrm{P}$ e K, respectivamente. Portanto, a necessidade de complementação nutricional foi de $\mathrm{N}=4,89 ; \mathrm{P}=7,84 ; \mathrm{K}=14,44$ (g/planta). A estimativa do fornecimento total de nutrientes às plantas (substrato mais doses) é apresentada na Tabela 1 .

Os frutos foram colhidos aos 70 dias após o transplantio (DAT), contados e pesados em balança digital. As características de produção avaliadas foram: produtividade de frutos (PT) representada por produção média de frutos por área, expressa em $\mathrm{t} / \mathrm{ha}$, e acumulada nas diferentes etapas de colheita; diâmetro de frutos (DT) obtido pela medição em paquímetro digital; e massa média de frutos (PM) pesados em balança digital nas diferentes datas de colheita por parcela.

As análises do teor de sólidos solúveis, expressos em ${ }^{0}$ Brix, $\mathrm{pH}$ e acidez titulável foram realizadas ao final do experimento. Os frutos foram colhidos ao acaso, separados e macerados em liquidificador, para extração do suco. Para as determinações foram utilizados um refratômetro manual e um potenciômetro digital.

A acidez titulável foi determinada por titulação feita com solução de $\mathrm{NaOH}$ $0,1 \mathrm{~N}$, utilizando-se 5,0 g do suco, diluídos em 100 mL de água destilada, em três amostras, conforme recomendações do Instituto Adolfo Lutz (1985), sendo os resultados expressos em porcentagem de ácido cítrico.

Para a resistência da polpa, foram escolhidos dois frutos maduros ao acaso, por unidade experimental, medidos individualmente. Os dados foram obtidos calculando-se a média de dois pontos, através do Texturômetro Stevens-LFRA Texture Analyser USA, com ponta de prova TA 9/1000, e velocidade de penetração de $15 \mathrm{~mm} / \mathrm{s}$, determinando-se a força de resistência à compressão na condição de retorno ao início.

Os dados de natureza qualitativa foram submetidos à análise de variância pelo teste F e teste de Tukey. Os dados de natureza quantitativa foram submetidos à análise de variância e, quando verificado o nível de significância de 1 e $5 \%$ foram testados os modelos linear, logarítmico, exponencial e o polinomial quadrático. Para a comparação do grupo fatorial com testemunha adicional, aplicou-se o teste de Dunnett a 5\% de probabilidade.

As equações que melhor se ajustarem aos dados foram selecionadas com base no coeficiente de determinação $\left(\mathrm{R}^{2}\right)$. As análises estatísticas foram realizadas com o auxílio dos aplicativos Microsoft Office Excel (2003), por meio do software ASSISTAT.

\section{RESULTADOS E DISCUSSÃO}

O biofertilizante misto aumentou a massa média de frutos em relação ao biofertilizante bovino nas doses 1,5 e 2,0 L/planta/semana, com valores de 1,52 e $1,44 \mathrm{~kg}$, respectivamente (Tabela $2)$. Esses valores também foram maiores que os observados nos tratamentos controle $(0,99 \mathrm{~kg})$ e adubação mineral exclusiva $(1,02 \mathrm{~kg})$.

Os valores mostrados se encontram superiores aos registrados por Silva et al. (2009) que, avaliando o efeito de fosfatos naturais no cultivo de melão orgânico, verificaram pesos médios variando entre 1,23 e 1,41 kg; e sob adubação mineral com supertriplo média de $1,45 \mathrm{~kg}$.

A massa média de frutos apresentou aumento linear em função das doses de biofertilizante bovino (Figura 1A). No entanto, o modelo polinomial quadrático se ajustou melhor com o uso do biofetilizante misto. No geral ocorreram valores maiores com o uso do misto (B1), sendo explicado principalmente pela sua maior riqueza nutricional. Esse maior aporte nutricional possibilita maiores taxas fotossintéticas e, consequentemente, maiores quantidades de carboidratos que são alocados para os órgãos de reserva das plantas evidenciando frutos mais pesados.

O aumento da massa média de frutos com a elevação da dosagem mostra que a diversidade de nutrientes essenciais presentes nos biofertilizantes contribuiu para o aumento da produtividade das culturas, semelhante ao observado por Freire et al. (2009) e Rodrigues et al. (2008).

Entretanto, outros autores não encontraram diferença no peso médio dos frutos com o uso de adubação orgânica. Silva et al. (2003), avaliando o efeito da aplicação de matéria orgânica diretamente no solo e de $\mathrm{N}$ via fertirrigação na cultura do melão híbrido AF 682 , verificaram que o peso médio dos frutos não foi influenciado por nenhum tratamento, variando de 1,63 a $1,79 \mathrm{~kg}$, respectivamente. Do mesmo modo, Freire et al. (2009), estudando o efeito da aplicação de doses do composto orgânico líquido (30, 60 e 90 L/ha/dia) na cultura do meloeiro, também não encontraram resposta significativa do peso médio dos frutos, tendo-se observado valores próximos de $2,5 \mathrm{~kg}$.

Com exceção apenas da dose $0,5 \mathrm{~L} /$ planta/semana, o biofertilizante misto possibilitou valores superiores aos observados com o bovino (Tabela 2). A maior quantidade de nutrientes presentes nesse biofertilizante possivelmente evidenciou esse resultado.

Valores semelhantes aos observados nesta pesquisa para o diâmetro do fruto foram verificados por Melo et al. (2012)

Tabela 1. Estimativa do fornecimento total de nutrientes no ciclo da cultura do melão (estimate of the total supply of nutrients for the melon crop). Fortaleza, UFC, 2011.

\begin{tabular}{|c|c|c|c|c|c|c|}
\hline \multirow{3}{*}{$\begin{array}{l}\text { Tratamentos } \\
\text { (L/semana/planta) }\end{array}$} & \multicolumn{6}{|c|}{ Acúmulo de nutrientes ${ }^{\#}$ (g/planta) } \\
\hline & \multicolumn{3}{|c|}{ Misto } & \multicolumn{3}{|c|}{ Bovino } \\
\hline & $\mathbf{N}$ & $\mathbf{P}$ & $\mathbf{K}$ & $\mathbf{N}$ & $\mathbf{P}$ & $\mathbf{K}$ \\
\hline $\mathrm{T} 1=0,5$ & 9,81 & 13,71 & 17,76 & 9,11 & 12,31 & 15,76 \\
\hline $\mathrm{T} 2=1,0$ & 11,96 & 17,76 & 25,76 & 10,41 & 14,86 & 21,76 \\
\hline $\mathrm{T} 3=1,5$ & 13,81 & 22,19 & 33,76 & 11,71 & 17,41 & 27,76 \\
\hline $\mathrm{T} 4=2,0$ & 15,81 & 26,36 & 41,76 & 13,01 & 19,96 & 33,76 \\
\hline
\end{tabular}

\#= Somatório da quantidade aplicado no substrato + quantidade aplicada por dose de biofertilizante (sum of the amount applied to the substrate + amount applied per biofertilizer dose). 
Tabela 2. Massa média de frutos, diâmetro transversal e produtividade de frutos de meloeiro submetido a tipos e doses de biofertilizantes em meloeiro (average fruit weight, transversal diameter and fruit yield of melon plants subjected to types and doses of biofertilizers). Fortaleza, UFC, 2011.

\begin{tabular}{|c|c|c|c|c|}
\hline \multirow{2}{*}{ Biofertilizantes } & \multicolumn{4}{|c|}{ Doses aplicadas (L/planta/semana) } \\
\hline & 0,5 & 1,0 & 1,5 & 2,0 \\
\hline & \multicolumn{4}{|c|}{ Massa média de frutos (kg/planta) } \\
\hline Misto & $1,19 \mathrm{a}$ & $1,31 \mathrm{a}$ & $1,52 \mathrm{a}$ & $1,44 \mathrm{a}$ \\
\hline \multirow[t]{2}{*}{ Bovino } & $1,11 \mathrm{a}$ & $1,25 \mathrm{a}$ & $1,20 \mathrm{~b}$ & $1,15 \mathrm{~b}$ \\
\hline & \multicolumn{4}{|c|}{ Tratamentos adicionais } \\
\hline Controle & & 0,99 & mineral & 1,02 \\
\hline \multirow[t]{2}{*}{$\mathrm{CV}(\%)$} & & & \multicolumn{2}{|c|}{13,20} \\
\hline & \multicolumn{4}{|c|}{ Diâmetro Transversal do fruto (cm) } \\
\hline Misto & $12,92 \mathrm{a}$ & $13,78 \mathrm{a}$ & $14,04 \mathrm{a}$ & $14,14 \mathrm{a}$ \\
\hline \multirow[t]{2}{*}{ Bovino } & $12,57 \mathrm{a}$ & $13,12 \mathrm{~b}$ & $13,02 \mathrm{~b}$ & $12,75 \mathrm{~b}$ \\
\hline & \multicolumn{4}{|c|}{ Tratamentos adicionais } \\
\hline Controle & & 12,15 & mineral & 12,05 \\
\hline \multirow[t]{2}{*}{$\mathrm{CV}(\%)$} & & & \multicolumn{2}{|c|}{3,73} \\
\hline & \multicolumn{4}{|c|}{ Produtividade (t/ha) } \\
\hline Misto & $28,16 \mathrm{a}$ & $33,51 \mathrm{a}$ & $29,58 \mathrm{a}$ & $22,83 \mathrm{a}$ \\
\hline \multirow[t]{2}{*}{ Bovino } & $18,43 \mathrm{~b}$ & $21,38 \mathrm{~b}$ & $28,58 \mathrm{a}$ & $21,43 \mathrm{a}$ \\
\hline & \multicolumn{4}{|c|}{ Tratamentos adicionais } \\
\hline Controle & & 18,73 & mineral & 20,40 \\
\hline CV $(\%)$ & & & & \\
\hline
\end{tabular}

Médias seguidas de mesma letra minúscula $(a, b)$ nas colunas não diferem entre si pelo teste de Tukey a $5 \%$ means followed by the same letter $(\mathrm{a}, \mathrm{b})$ in columns do not differ by Tukey test at $5 \%$.

os quais, utilizando fibra de coco como substrato no cultivo de melão rendilhado em cultivo protegido, obtiveram diâmetro transversal de $14,08 \mathrm{~cm}$. Contrariando os resultados observados neste experimento, Costa et al. (2011), avaliando o efeito da adubação suplementada com diferentes adubos orgânicos (esterco bovino, caprino e ovino) na cultura do meloeiro, não encontraram diferenças para essa variável.

As variações do diâmetro dos frutos em função das doses de biofertilizantes aplicadas também foram explicadas pelo modelo linear, para os biofertilizantes misto (B1) e bovino (B2), respectivamente. O B1 apresentou melhor desempenho, registrando um diâmetro máximo $(14,13 \mathrm{~cm})$ para uma dose de 2,0 L/planta/semana, com tendência crescente. Já no B2 houve diminuição do diâmetro com a elevação das doses (Figura 1B).

O biofertilizante misto induziu resultados superiores aos obtidos com o uso do biofertilizante bovino nas menores doses (Tabela 2), sendo que a maior produtividade média $(33,5 \mathrm{t} / \mathrm{ha})$ foi obtida na dose 1,0 L/planta/semana.

Contrariando esse estudo, Mesquita et al. (2007) observaram superioridade do biofertilizante bovino simples que proporcionou produtividade média de $53,5 \mathrm{t} / \mathrm{ha}$, enquanto o biofertilizante bovino enriquecido com macro, miuma produtividade de 50,1 t/ha. Por outro lado, Freire et al. (2009), estudando o efeito da aplicação de doses do composto orgânico líquido $(30,60$ e 90 $\mathrm{L} / \mathrm{ha} /$ dia) na cultura do meloeiro, não encontraram resposta significativa para essa característica.

É importante salientar que a produtividade média obtida por produtores tradicionais é de 30 t/ha, sendo que Costa et al. (2011) encontraram produtividade de 45 e 40 t/ha com os adubos orgânicos ovinos e caprinos, respectivamente, para o melão Cantaloupe.

Na Figura 1C, constatam-se variações da produtividade em função das cronutrientes e mistura protéica gerou doses de biofertilizantes aplicadas, sendo que o modelo quadrático explicou a relação para os biofertilizantes misto (B1) e bovino (B2). Apresentando o biofertilizante misto com máxima de $32,62 \mathrm{t} / \mathrm{ha}$, na dose de $1,08 \mathrm{~L} /$ planta/ semana, e o biofertilizante bovino com máxima de 25,87 t/ha, na dose 1,41 L/ planta/semana. A maior produtividade obtida com B1, além da maximização com menor dose, refletem a sua superioridade nutricional (Tabela 1).

Estudos com biofertilizantes e fontes orgânicas evidenciam resultados interessantes quando comparados ao presente estudo. A exemplo, Freire et al. (2009) verificaram uma produtividade superior à desse estudo para a cultura do meloeiro $(52,0 \mathrm{t} / \mathrm{ha})$ ao aplicar composto orgânico líquido via fertirrigação. Oliveira et al. (2011), avaliando a cultura da batatinha em solo adubado com adubação orgânica e mineral, observaram melhor desempenho de produtividade com fertilizante mineral. Com a cultura da abobrinha híbrida, Santos et al. (2012) constataram que a maior produtividade $(7,54 \mathrm{t} / \mathrm{ha})$ foi alcançada na dose de $35,34 \mathrm{~L} / \mathrm{ha}$ de biofertilizante suíno.

É pertinente ressaltar que a influência positiva de fontes orgânicas aplicadas via solo sobre aspectos produtivos de culturas agrícolas está relacionado ao efeito nutricional e consequentemente sobre os índices fisiológicos. Larcher (2006) afirma que a influência do estado nutricional da planta sobre as trocas gasosas, em especial a fotossíntese, ocorre de muitas maneiras, sendo que quase sempre maiores taxas fotossintéticas são alcançadas por meio da adubação. Ainda, a respeito da fotossíntese, Taiz \& Zeiger (2009) reforçam que essa troca gasosa tem recebido especial atenção por ser a principal fonte de carbono orgânico, de energia para o crescimento, produção de biomassa das plantas e produtividade.

Para as características de pós-colheita, com exceção da firmeza da polpa e dos sólidos solúveis (SS), as interações entre os fatores tipos e doses de biofertilizantes foram significativas. O B1 possibilitou média superior da firmeza $\left(11,84 \mathrm{gf} / \mathrm{cm}^{2}\right)$ à obtida com o B2 (11,18 gf/ $\left.\mathrm{cm}^{2}\right)$ (Figura 2a).

Essa variável é um dos recursos mais 
Tabela 3. Teste de comparação de médias da espessura do mesocarpo, da cavidade da polpa e da acidez titulável do meloeiro submetido a tipos e doses de biofertilizantes (comparison of average thickness of the mesocarp pulp cavity and titratable acidity of melon subjected to types and doses of biofertilizers). Fortaleza, UFC, 2011.

\begin{tabular}{|c|c|c|c|c|}
\hline \multirow{2}{*}{ Biofertilizantes } & \multicolumn{4}{|c|}{ Doses aplicadas (L/planta/semana) } \\
\hline & 0,5 & 1,0 & $\mathbf{1 , 5}$ & 2,0 \\
\hline & \multicolumn{4}{|c|}{ Espessura da polpa $(\mathrm{cm})$} \\
\hline Misto & $4,12 \mathrm{a}$ & $3,92 \mathrm{a}$ & $4,29 \mathrm{a}$ & $4,17 \mathrm{a}$ \\
\hline \multirow[t]{2}{*}{ Bovino } & $4,09 \mathrm{a}$ & $4,18 \mathrm{a}$ & $4,26 \mathrm{a}$ & $3,84 \mathrm{~b}$ \\
\hline & \multicolumn{4}{|c|}{ Tratamentos adicionais $(\mathrm{cm})$} \\
\hline Controle & & 3,83 & mineral & 3,86 \\
\hline \multirow[t]{2}{*}{$\mathrm{CV}(\%)$} & & & \multicolumn{2}{|c|}{10,67} \\
\hline & \multicolumn{4}{|c|}{ Cavidade da polpa $(\mathrm{cm})$} \\
\hline Misto & $4,84 \mathrm{a}$ & $5,20 \mathrm{a}$ & $5,63 \mathrm{a}$ & $5,81 \mathrm{a}$ \\
\hline \multirow[t]{2}{*}{ Bovino } & $4,99 \mathrm{a}$ & $4,94 \mathrm{a}$ & $5,16 \mathrm{~b}$ & $5,03 \mathrm{~b}$ \\
\hline & \multicolumn{4}{|c|}{ Tratamentos adicionais } \\
\hline Controle & & 47,35 & mineral & 43,68 \\
\hline \multirow[t]{2}{*}{$\mathrm{CV}(\%)$} & & & \multicolumn{2}{|c|}{8,2} \\
\hline & \multicolumn{4}{|c|}{ Acidez titulável (\% de ácido cítrico) } \\
\hline Misto & $0,14 \mathrm{a}$ & $0,20 \mathrm{a}$ & $0,18 \mathrm{~b}$ & $0,13 \mathrm{~b}$ \\
\hline \multirow[t]{2}{*}{ Bovino } & $0,12 \mathrm{a}$ & $0,20 \mathrm{a}$ & $0,21 \mathrm{a}$ & $0,21 \mathrm{a}$ \\
\hline & \multicolumn{4}{|c|}{ Tratamentos adicionais } \\
\hline Controle & & 0,21 & mineral & 0,2 \\
\hline $\mathrm{CV}(\%)$ & & & & \\
\hline
\end{tabular}

Médias seguidas de mesma letra minúscula $(a, b)$ nas colunas não diferem entre si pelo teste de Tukey a $5 \%$ (means followed by the same letter $(a, b)$ in columns do not differ by Tukey test at 5\%).

utilizados no acompanhamento da qualidade dos frutos (Hobson \& Grienson, 1993), sendo importante para definição de mercado, pois frutos mais firmes são mais resistentes às injúrias mecânicas durante o transporte e a comercialização no mercado exportador. Por conseguinte, a maior firmeza dos frutos produzidos com B1, possibilita aos frutos produzidos com este biofertilizante maior vida útil pós-colheita.

É pertinente ressaltar que o B1 apresenta maior teor de Ca em sua composição. Sendo um dos mais importantes nutrientes para as cucurbitáceas, estando o mesmo associado com a firmeza da polpa, melhorando a condição para armazenamento. Segundo Paiva et al. (2009), a pectina é um polissacarídeo, componente multifuncional na parede celular dos vegetais, participando da manutenção da união intercelular, juntamente com a celulose e a hemicelulose. Ainda, segundo os autores, o pectato de cálcio é de suma importância nas al. (2008), estudando cinco cultivares de melão rendilhado cultivados no solo e em substrato de fibra da casca de coco, também obtiveram menor espessura de polpa $(3,78 \mathrm{~cm})$.

As diferentes doses de biofertilizantes aplicadas ao solo implicaram em variações quadráticas quanto da espessura da polpa para B1 e B2 (Figura 2B). A máxima espessura da polpa foi de $4,32 \mathrm{~cm}$ na dose $1,12 \mathrm{~L} /$ planta/ semana do biofertilizante bovino (B1). Esta espessura foi superior à máxima obtida com B2, 4,26 cm na dose 1,48 L/planta/semana.

Provavelmente, as maiores dosagens ocasionaram excessos de nutrientes essenciais e de sódio, podendo ter contribuído para um efeito antagônico e salino nas plantas. Como os biofertilizantes apresentaram teores elevados de sódio, pode ter ocorrido um efeito antagônico com o potássio. De forma similar, Frizzone et al. (2005), em trabalho realizado em ambiente protegido com a cultura do melão, constataram que as doses elevadas de potássio aplicadas via fertirrigação afetaram a espessura da polpa, registrando um valor máximo de $2,29 \mathrm{~cm}$.

O teste de comparação de médias para a cavidade de polpa (Tabela 3) mostrou que o B1 apresentou valores médios maiores que o $\mathrm{B} 2$ nas doses 1,5 L/planta/semana e 2,0 L/planta/semana. Provavelmente o menor poder nutricional do biofertilizante bovino induziu maiores cavidades. Cabe ressaltar que, cavidades maiores tendem a reduzir a vida útil dos frutos e Rizzo \& Braz (2004) afirmam que menores valores da cavidade interna estão associados à melhor qualidade dos frutos, podendo ocorrer o desprendimento das sementes e a indesejada fermentação dos frutos.

Queiroga et al. (2010), avaliando o efeito da adubação mineral com ácido bórico na qualidade de frutos do híbrido de melão 'Florentino' pertencente ao grupo cantaloupensis, observaram valores de cavidade de polpa superior ao desse estudo $(5,85 \mathrm{~cm})$.

A cavidade da polpa variou linearmente em função das doses de biofertilizante, observando-se que o B1 apresentou dados médios superiores 


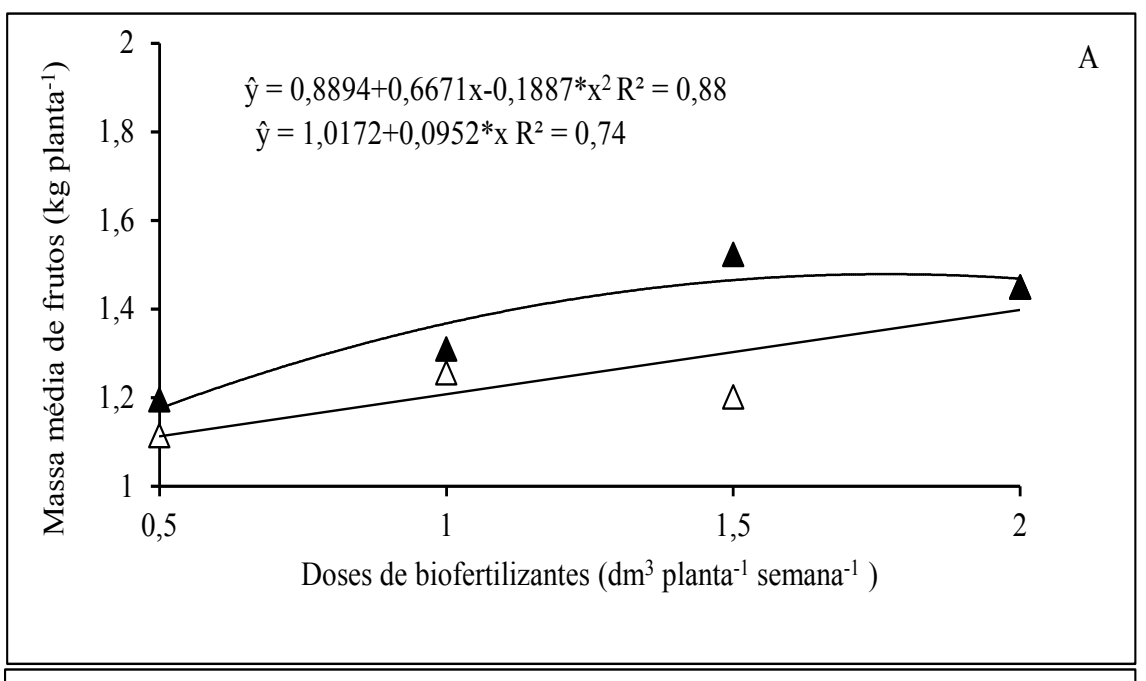

B
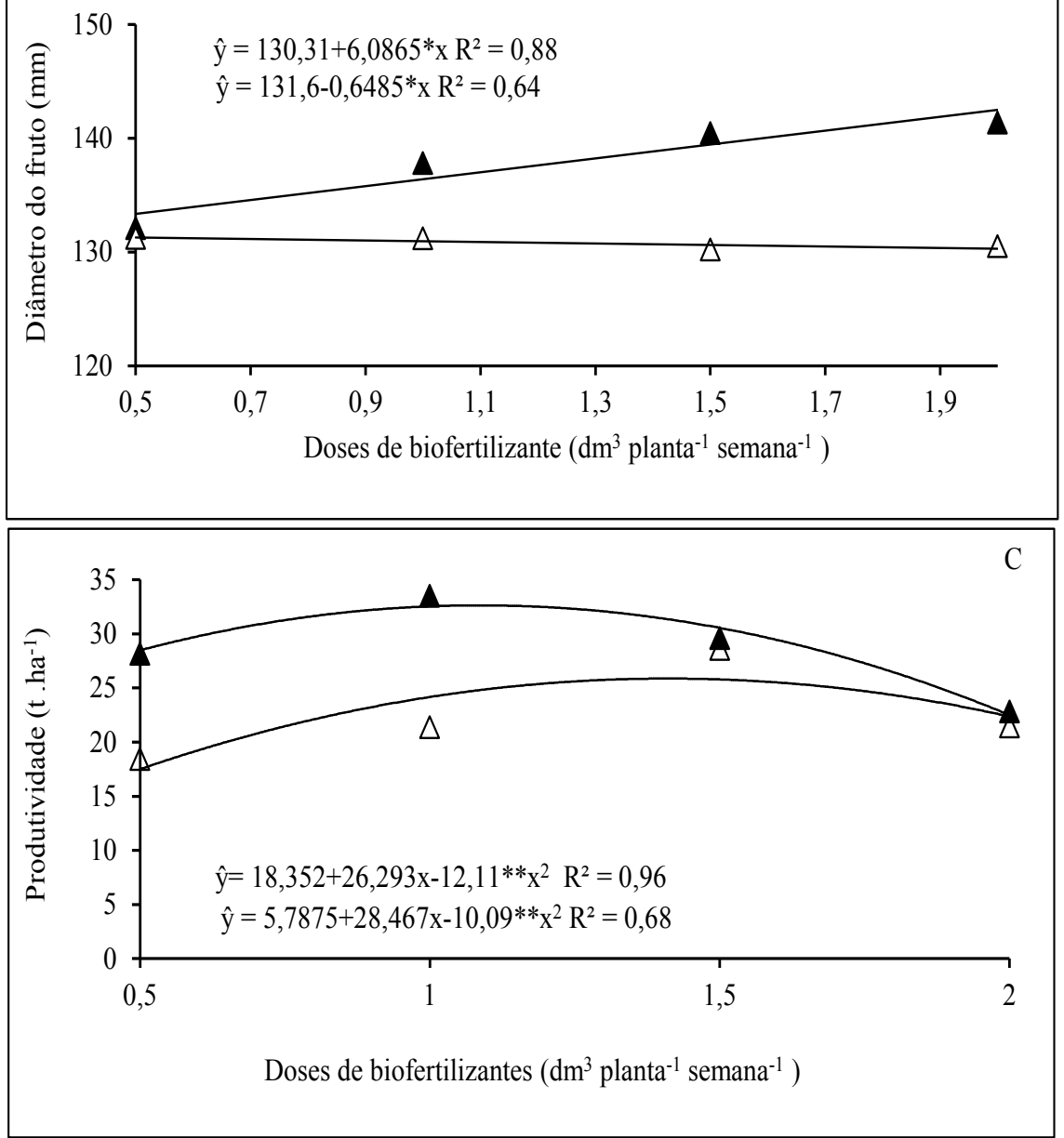

Figura 1. Peso médio (A), diâmetro transversal (B) e produtividade de frutos $(\mathrm{C})$ de melão submetido a tipos e doses de biofertilizantes $(\mathrm{B} 1(\boldsymbol{\Lambda})$ misto e B2 $(\triangle)$ bovino) [average weight (A), transversal diameter (B) and fruit yield (C) of melon subjected to types and doses of biofertilizers (B1 $(\boldsymbol{\Lambda})$ mixed and B2 $(\triangle)$ bovine)]. Fortaleza, UFC, 2011.

ao B2 (Figura 2C), o que comprova o fato anterior: menor fertilidade, menor qualidade de frutos. Em oposição ao obtido neste trabalho, Pinto et al. (2008), aplicando biofertilizante e substâncias húmicas via fertirrigação no cultivo de meloeiro, observaram que não houve efeito significativo sobre essa variável.

Os valores médios para a acidez titulável revelam que o B2 proporcio- nando resultados superiores aos de B1 nas doses 1,5 e 2,0 L/planta/semana, chegando à média de $0,21 \%$, semelhante ao tratamento controle e à adubação mineral (Tabela 3). Esse resultado pode estar associado ao tipo de fermentação e aos componentes de preparo dos biofertilizantes. Prado (2008) afirma que uma das características para uma elevada porcentagem de AT em frutos diz respeito à nutrição fornecida às culturas.

Santos et al. (2011) constataram aumento da acidez titulável da cultivar "Hope King" de melão rendilhado ao utilizar esterco caprino como fonte orgânica. No entanto, esses mesmos autores alertam que a perda de acidez é considerada desejável em grande parte dos frutos, sendo importante para o processo de amadurecimento, onde são provavelmente convertidos em açúcares. Em oposição ao obtido neste trabalho, Pinto et al. (2008), em pesquisa realizada no distrito de irrigação Senador Nilo Coelho, Petrolina-PE, aplicando biofertilizante e substâncias húmicas via fertirrigação no cultivo de meloeiro, observaram que não houve efeito significativo para essa variável. Mesquita et al. (2007), analisando o efeito da aplicação de biofertilizante simples e enriquecido, encontraram valores maiores ao desse estudo para essa variável (6\%) ao aplicar 2,0 L/cova na cultura do mamoeiro durante o primeiro ciclo produtivo.

Na Figura 2D observa-se um modelo linear para a acidez titulável sob as diferentes doses de biofertilizante, sendo crescente para o B1 e decrescente para B2. Sabe-se que, para uma boa qualidade de frutos, é interessante que os teores de ácidos orgânicos estejam baixos, para que o ratio, relação sólidos solúveis e acidez titulável, alcance valores que indiquem boa palatabilidade do fruto.

Em oposição a esses resultados, Melo et al. (2012), cultivando o melão Bônus $n^{\circ} 2$, Louis e Fantasy em diferentes substratos de fonte orgânica (fibra de coco e casca de amendoim), verificaram que não houve efeito significativo. Já Mesquita et al. (2007), ao analisar o efeito do biofertilizante simples na dose 2,0 L/cova, também obtiveram respostas lineares em plantas de mamoeiro.

O biofertilizante misto mostrou- 

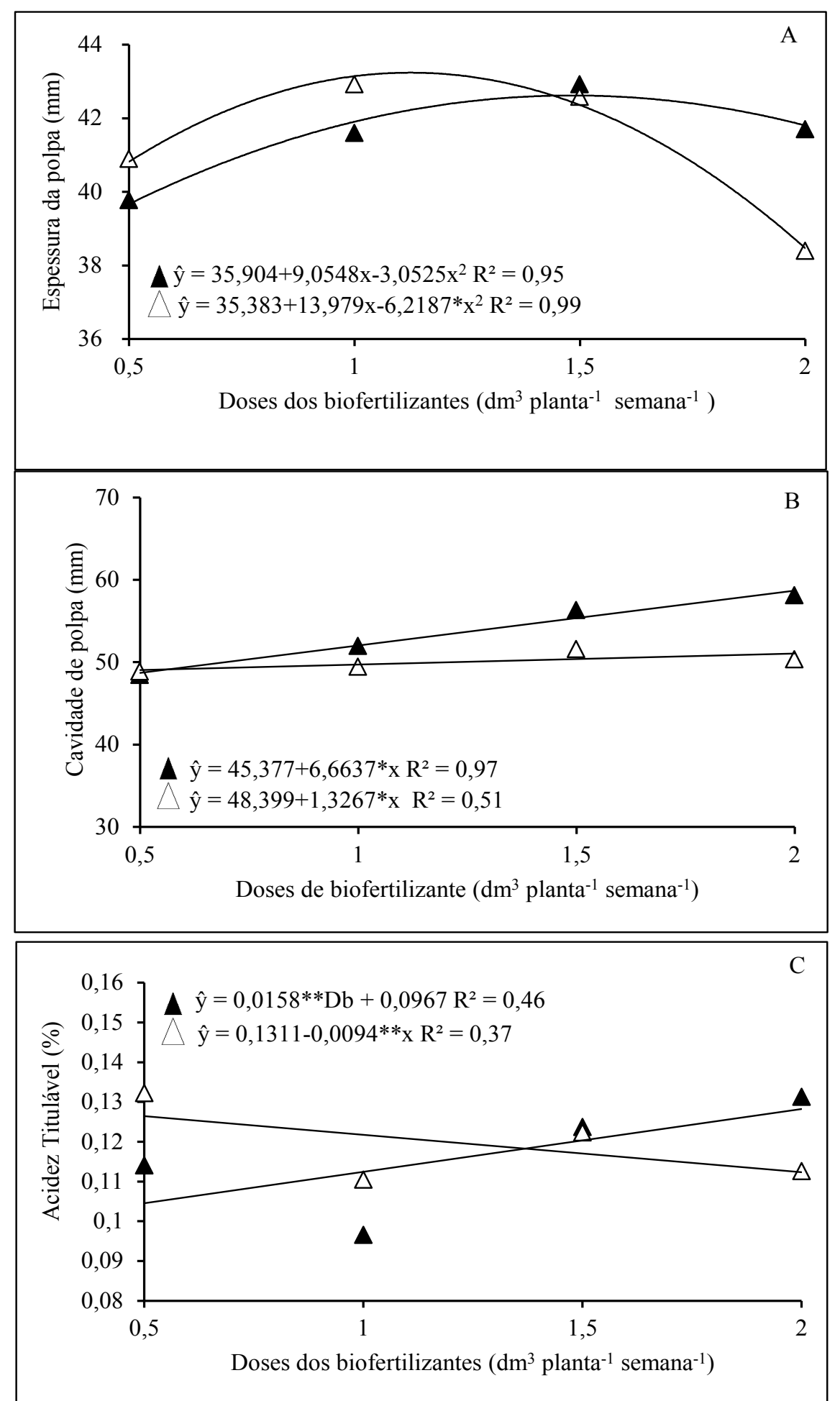

Figura 2. Firmeza de polpa (A) espessura da polpa (B), cavidade da polpa (C) e acidez titulável (D) de melão submetido a tipos e doses de biofertilizantes: B1 $(\boldsymbol{\Lambda})$ misto e B2 $(\triangle)$ bovino [pulp firmness (A) pulp thickness (B), pulp cavity (C) and titratable acidity (D) of melon fruits subjected to types and doses of biofertilizers: B1 $(\boldsymbol{\Delta})$ mixed and B2 $(\Delta)$ bovine]. Fortaleza, UFC, 2011.

-se mais eficiente do que o bovino na maioria das variáveis analisadas. A maior produtividade do meloeiro $(32,62$ $\mathrm{t} / \mathrm{ha}$ ) foi alcançada com a dose de 1,08 $\mathrm{L} /$ planta'semana para o biofertilizante vidade de polpa.

\section{REFERÊNCIAS}

CAVALCANTE LF; SANTOS GD; OLIVEIRA FA; CAVALCANTE IHL; GONDIM SC; CAVALCANTE MZB. 2007. Crescimento e produção do maracujazeiro amarelo em solo de baixa fertilidade tratado com biofertilizantes líquidos. Revista Brasileira de Ciências Agrárias 2: 15-19.

COSTA CC; SANTOS MF; LIMA PS; LOPES KP; SILVA RMB. 2011. Efeito da adubação de plantio suplementada com adubos orgânicos na produção do melão cantaloupe. Horticultura Brasileira 29: 4026-4033.

EMBRAPA. 2006. Sistema brasileiro de classificação de solos. Rio de Janeiro: Embrapa Solos. 306p.

FAO. 2012. Faostat. Disponível em http:// faostat. fao.org/site/567/DesktopDefault. aspx?PageID=567\#ancor. Acessado em 24 abr. 2014.

FREIRE GM; MEDEIROS JF; OLIVEIRA FA; AMÂNCIO MG; PONTES NC; SOARES IAA; SOUZA ALM. 2009. Aplicação de composto orgânico líquido via fertirrigação na cultura do meloeiro. Bioscience Journal, 25: 49-55.

FRIZZONE JA; CARDOSO SS; REZENDE R. 2005. Produtividade e qualidade de frutos de meloeiro cultivado em ambiente protegido com aplicação de dióxido de carbono e de potássio via água de irrigação. Acta scientiarum, .27: 707-717.

HOBSON GE; GRIERSON D. 1993. Tomato. In: SEYMOUR GB; TAYLOR JE; TUCKER GA (eds). Biochemistry of fruit ripening. London: Chapman \& Hall. p. 405-442.

IAL. 1985. Normas analíticas, métodos químicos e físicos para análise de alimentos. São Paulo: Instituto Adolfo Lutz. 533p.

IBGE. 2012. Produção Agrícola. Disponível em: http://www.ibge. gov.br/estadosat/temas.php ?sigla $=$ pe\&tema=lavouratemporaria.2012>. Acessado em 22 abr. 2014.

LARCHER W. 2006. Ecofisiologia vegetal. São Carlos: Rima Artes e Textos. 550p.

MELO DM; CASTOLDI R; CHARLO HCO; GALATTI FS; BRAZ TL. 2012. Produção e qualidade de melão rendilhado sob diferentes substratos em cultivo protegido. Revista Caatinga 25: 58-66.

MESQUITA EF; CAVALCANTE LF; GONDIM SC; CAVALCANTE IHL; ARAÚJO FAR; BECKMANN-CAVALCANTE MZ. 2007. Produtividade e qualidade de frutos do mamoeiro em função de tipos e doses de biofertilizantes. Semina, 28: 589-596.

OLIVEIRA FF; SALCEDO IHE GALVÃO SRS. 2011. Adubação orgânica e inorgânica da batatinha em solos arenosos: Produtividade, nutrientes na planta e lixiviação. Revista Brasileira de Engenharia Agrícola e Ambiental 15: 1228-1234.

PAIVA EP; LIMA MS; PAIXÃO JA. 2009. Pectina: propriedades químicas e importância sobre a estrutura da parede celular de frutos 
durante o processo de maturação. Revista Iberoamericana de Polímero 10: 196-211.

PENTEADO SR 2007. Adubação orgânica: Compostos orgânicos e biofertilizantes. Campinas: Edição do autor. 162p.

PINTO JM; GAVA CAT; LIMA MAC; SILVA AF; RESENDE GM. 2008. Cultivo orgânico do meloeiro com aplicação de biofertilizantes e doses de substância húmica via fertirrigação. Revista Ceres 55: 280-286.

PRADO RM. 2008. Nutrição de plantas. São Paulo: Unesp. 407p.

QUEIROGA FM; COSTA SA; PEREIRA FHF; MARACAJÁ PB; SOUSA FILHO AL. 2010. Efeito de doses de ácido bórico na produção e qualidade de frutos de melão Harper. Revista Verde 5: 132-139.

QUEIROGA RCF; PUIATTI M; FONTES PCR; CECON PR. 2008. Produtividade e qualidade do melão cantaloupe, cultivado em ambiente protegido, variando o número e a posição dos frutos na planta. Bragantia, 67: 911-920.

RIZZO AAN; BRAZ LT. 2004. Desempenho de linhagens de melão rendilhado em casa de vegetação. Horticultura Brasileira 22:
784-788.

RODRIGUES AC; CAVALCANTE LF; DANTAS TAG; CAMPOS VB; DINIZ AA. 2008.

Caracterização de frutos de maracujazeiroamarelo em solo tratado com "biofertilizante Supermagro" e potássio. Revista Magistra 20: 264-272.

SANTOS ACV. 1992. Biofertilizantes líquidos: defensivo da natureza. Rio de Janeiro: Emater. $16 \mathrm{p}$.

SANTOS AF; COSTA CC; SILVA FVG; SILVA RMB; MEDEIROS LL. 2011. Qualidade de melão rendilhado sob diferentes doses nutricionais. Revista Verde 6: 134-145.

SANTOS MR; SEDIYAMA MAN; MOREIRA MA; MEGGUER CA; VIDIGAL SM. 2012. Rendimento, qualidade e absorção de nutrientes pelos frutos de abóbora em função de doses de biofertilizante. Horticultura Brasileira 30: 160-167.

SILVA DJ; FARIA CMB; PINTO JMP; COSTA ND; GAVA CAT; DIAS RCS; GOMES TCA; ARAÚJO JLP. 2009. Cultivo de melão orgânico: fosfatos naturais como fontes alternativas de fósforo. Revista Brasileira de Fruticultura 31: 559-566.

SILVA IR; MENDONÇA ES. 2007. Matéria orgânica do solo. In: NOVAIS RF; ALVAREZ VVH; BARROS NF; FONTES RLF; CANTARUTTI RB; NEVES JCL. (eds). Fertilidade do Solo. Viçosa: Sociedade Brasileira de Ciência do Solo, p. 275-374.

SILVA MA; CHAVES LHG; SILVA DJ; FARIA AF. 2003. Produtividade e qualidade do melão em função de nitrogênio, micronutrientes e matéria orgânica. Agropecuária Técnica 24: 131-138.

TAIZ L; ZEIGER E. 2009. Fisiologia vegetal. Porto Alegre: ARTMED.729p.

TERCEIRO NETO CPC; MEDEIROS JF; GHEYI HR; DIAS NS; OLIVEIRA FRA; LIMA KS. 2012. Acúmulo de matéria seca e nutrientes no meloeiro irrigado sob estratégias de manejo da salinidade. Revista Brasileira de Engenharia Agricola e Ambiental 16: 1069-1077.

VARGAS PF; CASTOLDI R; CHARLO HCO; BRAZ LT. 2008. Qualidade de melão rendilhado (Cucumis melo) em função do sistema de cultivo. Ciência e Agrotecnologia 32: $137-142$. 